\title{
DISPOSITIVO DE PROTEÇÃO APÓS REPARAÇÃO DAS RUPTURAS DO MECANISMO EXTENSOR DO JOELHO
}

\author{
PROTECTION DEVICE ON THE REPAIR OF RUPTURES \\ OF KNEE EXTENSOR MECHANISM
}

\author{
Carlos Francisco Arguello Frutos ${ }^{1}$, \\ Osmar Pedro Arbix Camargo², \\ Nilson Roberto Severino ${ }^{3}$, \\ Ricardo de Paula Leite Cury ${ }^{4}$, \\ Victor Marques de Oliveira ${ }^{5}$, \\ Tatsuo Aihara ${ }^{5}$, \\ Roger Avakian 5
}

\section{RESUMO}

Objetivo: Avaliar os resultados obtidos com a realização da técnica de dispositivo de proteção nos pacientes submetidos à osteossíntese ou sutura de lesões do mecanismo extensor. Métodos: Foram avaliados 18 prontuários de pacientes que sofreram lesões traumáticas do aparelho extensor interessando a região entre a tuberosidade anterior da tíbia (TAT) e o ápice da patela submetido à técnica de dispositivo de proteção. A idade variou de 22 a 69 anos, com média de 44 anos. Houve predomínio do sexo masculino em $67 \%$ dos casos. O local mais acometido em $83 \%$ dos casos foi o $1 / 3$ apical da patela. Foi elaborado um protocolo para coleta dos dados informativos relacionando os pacientes e a sua evolução obtida em seus prontuários. Resultados: Observamos a consolidação da fratura de patela em todos os 17 pacientes, e a cicatrização do ligamento patelar num único caso. A dor esteve presente em quatro pacientes, sendo que não observamos nenhuma outra complicação relacionada com a técnica cirúrgica. Conclusão: $\mathrm{O}$ dispositivo de proteção é eficiente quando aplicado no tratamento operatório das lesões que se localizam entre o ápice da patela e a TAT propiciando movimentação ativa e passiva no pós-operatório imediato.

Descritores - Joelho; Ligamento patelar; Aavaliação de resultados

\section{ABSTRACT}

Objective: To evaluate results obtained using the protection device technique for osteosintesis or suture of extensor mechanism lesions. Material and Methods: The authors reviewed 18 charts of patients submitted to protection device technique due to traumatic lesion of extensor mechanism that had occurred between the anterior tibial tuberosity and the apical portion of patella. Age ranged from 22 to 69 years, with a mean of 44 years. Male patients prevailed, with $67 \%$ of the cases. The most affected spot was, in $83 \%$ of the cases, the apical distal third. A protocol was created to collect data, listing the patients and the clinical history from their medical records. Results: The authors observed consolidation of the patella fracture in all 17 patients, and cicatrization of the patellar ligament in one patient. Pain was described in four patients. There were no complications related to the procedure. Conclusion: The protection device showed to be efficient when used in surgical treatment of lesions between the apical patella and the anterior tibial tuberosity, providing active and passive mobility in the early postoperative time.

Keywords - Knee; Patellar ligament; Outcome assessment

1 - Médico Assistente do Grupo de Cirurgia do Joelho do DOT-FCMSCSP-SP

2 - Professor Adjunto, Coordenador dos Cursos de Graduação e Consultor do Grupo de Cirurgia do Joelho do DOT-FCMSCSP-SP

3 - Professor Assistente, Chefe do Grupo de Cirurgia do Joelho do DOT-FCMSCSP-SP

4 - Professor Instrutor do grupo do joelho do DOT-FCMSCSP-SP

5 - Médico Assistente do Grupo de Cirurgia do Joelho do DOT-FCMSCSP-SP

Trabalho realizado no Departamento de Ortopedia e Traumatologia da Faculdade de Ciências Médicas da Santa Casa de São Paulo, Pavilhão "Fernandinho Simonsen" (DOTFCMSCSP-SP), Diretor: Prof.Dr. Osmar Avanzi

Correspondência: Departamento de Ortopedia e Traumatologia ${ }^{-}$Irmandade da Santa Casa de Misericórdia de São Paulo - Rua Dr. Cesário Motta Jr. 112 - CEP 01221-020 - São Paulo, SP. Brasil. E-mail: dotjoelho@santacasasp.org.br

Declaração: Não houve auxílio, de qualquer espécie, a esta pesquisa e não há conflitos de interesse dos autores em relação ao presente manuscrito, conforme Resolução $\mathrm{n}^{\circ} 1.595 / 2000$ do Conselho Federal de Medicina. 


\section{INTRODUÇÃO}

O tratamento das lesões do aparelho extensor varia de acordo com a estrutura lesada, ou seja, o nível atingido. As rupturas decorrem de traumatismos e afecções degenerativas atingindo praticamente todas as faixas etárias. As fraturas da patela são as mais frequentes, sendo de incidência menor as lesões do tendão quadricipital e do ligamento patelar.

Do ponto de vista terapêutico, as fraturas distais da patela, atingindo o seu ápice, comportam-se e devem ser encaradas como avulsões do ligamento patelar, com tratamento cirúrgico se estão desviadas, sendo mais raras que as fraturas do corpo patelar ${ }^{(1)}$. A reinserção óssea ou tendinosa é sujeita as forças de tração muscular intensas, principalmente quando estimulamos a reabilitação precoce as quais podem levar à perda da redução e à diastase das estruturas reparadas pela tração excessiva frente à pouca resistência oferecida pelas mesmas.

Tanto na literatura como em nossa vida prática, são utilizados dispositivos que protegem a reparação, e procura-se realizá-los de forma que não tragam intercorrências que prejudiquem a recuperação do joelho. O modo de executar o dispositivo pode evitar tais intercorrências (em casos de lesão do aparelho extensor, a força de tensão pode superar a resistência mecânica tecidual, ou o material de sutura ou osteossintese utilizado, levando a novas roturas) e esse foi o motivo de desenvolvimento da técnica descrita neste trabalho.

\section{CASUÍSTICA E MÉTODOS}

Foram avaliados 18 prontuários de pacientes adultos, atendidos no Departamento de Ortopedia e Traumatologia da Santa Casa de São Paulo, Pavilhão "Fernandinho Simonsen", que sofreram lesões traumáticas do aparelho extensor interessando a região entre a tuberosidade anterior da tíbia (TAT) e o ápice da patela.

Após a reparação da lesão, com a finalidade de permitir a mobilização precoce do joelho foi utilizada a técnica do dispositivo de proteção descrita a seguir: após a reparação das estruturas rompidas, realiza-se com broca de $2 \mathrm{~mm}$ um orifício transversal no terço proximal a aproximadamente $1,5 \mathrm{~cm}$ da base da patela, no meio da sua espessura.

Passa-se um fio de arame com espessura 1,2mm.

As duas extremidades do fio entrecruzam sobre a patela e são tensionadas. Realiza-se então duas torceduras e o sistema com os dois fios são levados juntos até a TAT. Nessa região é introduzido um parafuso de cortical de $3,2 \mathrm{~mm}$, numa direção perpendicular ou oblíqua de distal a proximal à TAT fixando-o na cortical posterior tibial, porém deixando-o com o colo ainda saliente (Figura 1).
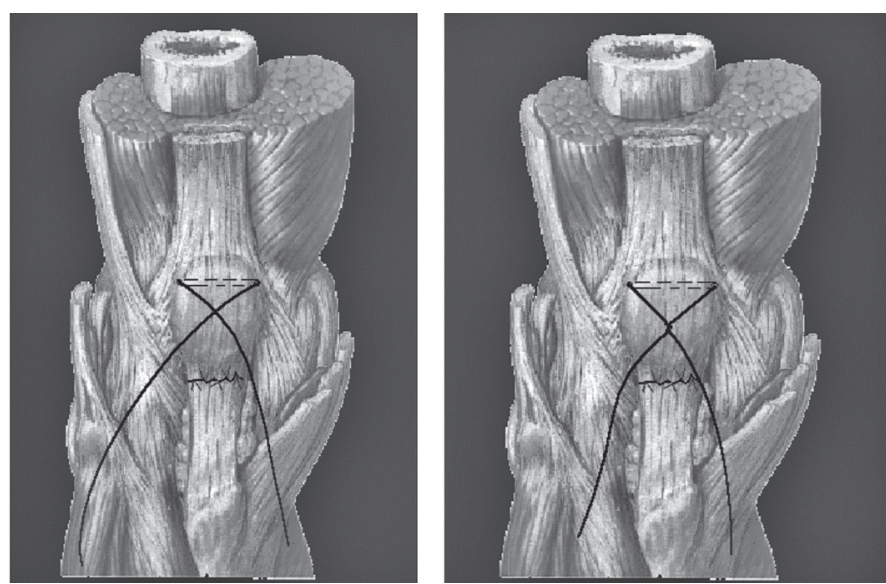

Figura 1 - Confecção do túnel na patela e passagem do fio de aço com sua torcedura proximal

Flexiona-se o joelho a $30^{\circ}$ (o ligamento patelar inicia a sua tensão a $30^{\circ}$ de flexão; de $0^{\circ}$ até $30^{\circ}$ existe uma frouxidão fisiológica com o quadríceps relaxado), marca-se nos fios o comprimento dos mesmos até o parafuso, e mais duas torceduras são realizadas.

As extremidades são enlaçadas em torno do colo do parafuso e é completada a fixação com as torceduras finais; nesse momento completa-se a introdução do mesmo, para que não fique saliente sob a pele (Figuras 2 e 3$)$.

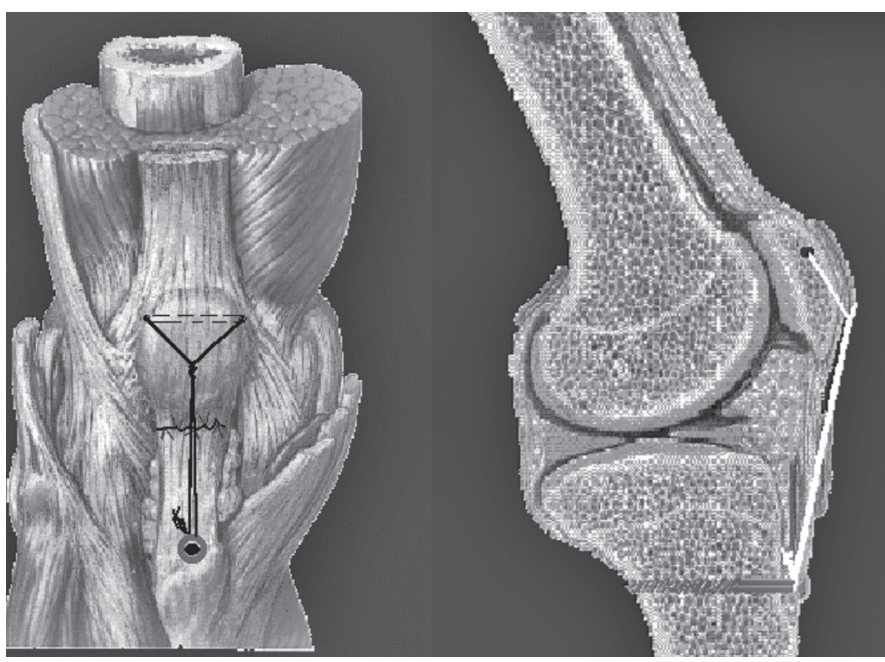

Figura 2 - Dispositivo de Proteção já ancorado no parafuso na TAT

Procede-se ao fechamento da incisão e, no pós-operatório, o paciente é estimulado a iniciar a flexo - extensão assistida pelo fisioterapeuta o qual inicia a movimentação passiva precoce e as contrações do quadríceps para a extensão ativa.

Foi elaborado um protocolo para coleta dos dados informativos relacionando os pacientes e a sua evolução obtida em seus prontuários (Anexo 1). 


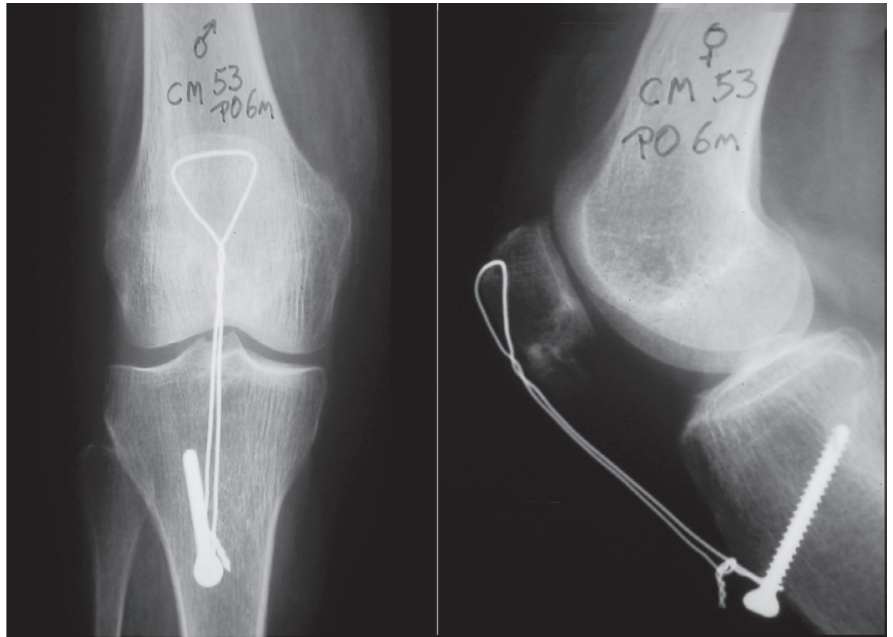

Figura 3 - Radiografias mostrando o DPD

\section{ANEXO 1}

PROTOCOLO DEAVALIAÇÃO DATÉCNICADE PROTEÇÃO DE OSTEOSSINTESE OU SUTURA DE LESÕES DO APARELHO EXTENSOR DO JOELHO

Nome:

$\mathrm{N}^{\circ}$ de prontuário: — Data de cirurgia

QUESTÕES:

LOCAL DA LESÃO:

DOR: $\operatorname{SIM}(\quad)$ NÃO( )

EXTENSÃO COMPLETA DO JOELHO: SIM（） NÃO（）

ALTURA DA PATELA: NORMAL（ ) ALTA（）BAIXA（）

NECESSIDADE DE RMS: SIM（） NÃO（）

CASO SIM, QUANTO TEMPO APÓS A CIRURGIA?

QUEBRA DE MATERIAL DE SÍNTESE DURANTE A EVOLUÇÃO:

SIM ( ) QUANTO TEMPO APÓS ?

NÃO ( )

\section{RESULTADOS}

Em nosso estudo foi observado que houve predominância do sexo masculino $(12=67 \%)$ em relação ao feminino $(6=33 \%)$. A média de idade foi de 44 anos variando de 22 a 84 anos.

Quanto a localização da lesão, em 83\% (15 pacientes) dos casos, o 1/3 distal apical foi comprometido por uma lesão simples, em 11\% (dois pacientes) houve uma fratura cominutiva do $1 / 3$ apical e em $6 \%$ (um paciente) o ligamento patelar foi comprometido na sua substância.

Para avaliação da dor após a fixação, a consideramos positiva quando ela estava presente e impossibilitava a flexo - extensão ativa de, no mínimo, além $90^{\circ}$; levando-se em consideração este critério, a dor estava presente em $22 \%$ dos casos (quatro pacientes). Em relação a extensão apenas um paciente $(6 \%)$ apresentou déficit de $-3^{\circ}$ (Tabela 1 ).
Tabela 1 - Evolução pós aplicação do DP

\begin{tabular}{l|c|c}
\hline TOTAL: 18 pacientes & SIM & $\%$ \\
\hline DOR & 4 & $22 \%$ \\
\hline PERDA FLEX̃̃O & 0 & $0 \%$ \\
\hline PERDA EXTENSÃO & 1 & $6 \%$ \\
\hline RMS $^{*}$ & 6 & $33 \%$ \\
\hline COMPLICAÇÕES $^{\prime}$ & 0 & $0 \%$ \\
\hline
\end{tabular}

${ }^{*}$ RMS-retirada de material de síntese

Houve necessidade de retirada de material de síntese (RMS) em seis pacientes (33\%), sendo esta realizada num período médio pós-operatório de oito meses. Dentre estes, ocorreu a quebra do material de síntese em 17\% (03 pacientes), que não interferiu na evolução do resultado final. Não tivemos nenhuma complicação relacionada com a técnica cirúrgica.

\section{DISCUSSÃO}

As rupturas do aparelho extensor decorrem de traumatismos e em alguns casos podem estar enfraquecidos por doenças sistêmicas e uso de esteróides ou fluoroquinolonas, atingindo praticamente todas as faixas etárias, interessando a patela, o ligamento patelar, a TAT e o tendão quadriciptal, e de tratamento cirúrgico, conforme relatam Mafulli et al ${ }^{(2)}$, Greis et $\mathrm{al}^{(3)}$, Hardy et $\mathrm{al}^{(4)}$, Rasul et al ${ }^{(5)}$.

Vários autores, entre os quais destacamos, Magnuson $^{\left({ }^{()}\right)}$, McConnell(7), Muller et al ${ }^{(\mathbf{( 8 )}}$, e Insall et $\mathrm{al}^{(\mathbf{9 )}}$ demonstraram uma sensível superioridade nos resultados obtidos quando se aplica uma banda de proteção ancorada em tecido sadio, ou seja, na porção íntegra da patela e na tuberosidade anterior da tíbia (TAT). Frisamos, contudo o relato de Enad et al ${ }^{(\mathbf{1 0 )}}$, que comparam os resultados funcionais e clínicos entre cinco pacientes submetidos a reabilitação precoce e cinco submetidos a imobilização e reabilitação tardia; não encontraram diferenças após 16 meses pós operatórios; concluíram que um estudo mais profundo e um tempo de evolução mais longo poderão evidenciar diferenças entre os dois métodos fisiátricos.

Essa banda tensora pode ser realizada utilizando-se tiras de fascia lata, fios de sutura não absorvíveis, próteses ligamentares, e arames de aço, com a forma circular, retangular ou em "oito" fixados na patela através de túneis na patela ou âncoras ${ }^{(11,12)}$. Em nosso Serviço temos utilizado com sucesso os fios de arame passados na forma de retângulo, ou em "oito" ancorados num parafuso que atravessa horizontalmente a TAT.

A técnica descrita utiliza-se de fios de aço por serem resistentes à tensão, e somente sofrerem ruptura por fadiga após três meses, tempo necessário para cicatrização 
das lesões; sendo que na grande maioria não trazem sintomatologia que justifiquem uma retirada precoce. Já nos casos em que tragam sintomas, ou nos atletas há a possbilidade da retirada logo após decorridos os três primeiros meses fundamentais para cicatrização definitiva tanto das lesões ligamentares, como ósseas. Um inconveniente seria a flexibilidade menor diante de fios não metálicos. Contudo, Kasten et $\mathbf{a l}^{(\mathbf{1 3})}$, relatam estudo comparativo entre os dois materiais concluindo pela superioridade dos arames, pois os fios sintéticos trouxeram complicações decorrentes de reações teciduais e infecção.

Concordamos com Bhargava et $\mathrm{al}^{(\mathbf{1 4 )}}$, Enad et $\mathrm{al}^{(\mathbf{1 5 )}}$, quando afirmam que o reparo primário protegido com fios de cerclagem, e, a mobilização precoce, propicia excelentes resultados no tratamento, tendo como vantagem diminuir o risco de artrofibrose, conseqüente limitação de movimentos e perda de função articular .

É comum os pacientes submetidos a esse tratamento queixarem-se de dor de intensidade variável, quando tentam flexionar o joelho, dificultando um dos objetivos que é recuperar rapidamente a amplitude de movimentos evitando-se aderências e retrações que bloqueiem uma flexão compatível com o joelho normal, obtendo-se resultados insatisfatórios.

Observamos que essa dor é causada pela pressão que o fio de arame exerce durante o movimento sobre o ligamento patelar, os retináculos, o coxim gorduroso retro patelar, a cápsula e membrana sinovial, contra os côndilos femorais, demovendo o paciente em prosseguir a fisioterapia.

A técnica de dispositivo de proteção segue exatamente a mesma direção do ligamento patelar, sem pressionar nenhuma estrutura, transferindo a força tensora do quadríceps diretamente à TAT.

Portanto, o dispositivo utilizado acompanha exatamente a mesma direção e sentido das forças tensoras

\section{REFERÊNCIAS}

1. Enad JG. Patellar tendon ruptures. South Med J. 1999;92(6):563-6.

2. Maffulli N, Wong J. Rupture of the achilles and patellar tendons. Clin Sports Med. 2003;22(4):761-76.

3. Greis PE, Lahav A, Holmstrom MC. Surgical treatment options for patella tendon rupture, part II: chronic. Orthopedics. 2005; 28(8):765-9.

4. Hardy JR, Chimutengwende-Gordon M, Bakar I. Rupture of the quadriceps tendon: an association with a patellar spur. J Bone Joint Surg Br. 2005;87(10):1361-63.

5. Rasul AT Jr, Fischer DA. Primary repair of quadriceps tendon ruptures. Results of treatment. Clin Orthop Relat Res. 1993; (289):205-7.

6. Magnuson PB. Fractures. 2nd ed. Philadelphia: JB Lippincott; 1933.

7. McConnell BE. An operation for fractures of the patella using the temporary patellar tendon substitution technic. South Med J. 1971; 64(1):87-9.

8. Muller ME, Allgower M, Schneider R, Willenegger H. Manual of Internal Fixation. 2nd ed. New York: Springer-Verlag; 1979.

9. Insall JN, Windsor RE, Scott WN, Kelly MA, Aglietti P. Cirurgia de la Rodilla. $2^{\text {a }}$ ed. Buenos Aires: Medica Panamericana; 1994. que agem num joelho normal durante a sua movimentação justificando a sua denominação como sendo uma proteção de efeito dinâmico.

Acreditamos que esse fato seria o responsável pelo menor número de queixas dolorosas nos pacientes avaliados neste estudo, o que facilitou a recuperação de movimentos considerada extremamente satisfatória.

Salientamos Shelbourne et $\mathrm{al}^{(\mathbf{1 6})}$, que utiliza esses mesmos princípios através do uso do cabo "Dall-Miles", um dispositivo metálico flexível fixado no centro da patela através de parafusos e com uma tensão regulável a $60^{\circ}$ de flexão; a sua retirada ocorreu entre a sexta e oitava semana. Em seus 10 pacientes conseguiu resultados semelhantes aos nossos, advogando que a posição do dispositivo, semelhante ao dispositivo de proteção permite uma imediata reabilitação com a restauração precoce de uma amplitude de movimentos satisfatória, permitindo a recuperação da força original do quadríceps. Esse dispositivo encerra dificuldades de sua utilização em nosso meio referentes ao seu custo, não trazendo vantagens ao dispositivo de proteção quanto aos nossos objetivos.

\section{CONCLUSÃO}

O dispositivo de proteção é eficiente quando aplicado no tratamento operatório das lesões que se localizam entre o ápice da patela e a TAT propiciando movimentação ativa e passiva no pós-operatório imediato, com um índice mínimo de queixas dolorosas.

\section{AGRADECIMENTO}

Agradecemos ao Núcleo de Apoio à Publicação da Faculdade de Ciências Médicas da Santa Casa de São Paulo - NAP-SC o suporte técnico-científico à publicação deste manuscrito.

10. Enad JG, Loomis LL. Patellar tendon repair: postoperative treatment Arch Phys Med Rehabil. 2000;81(6):786-8.

11. Bushnell BD, Byram IR, Weinhold PS, Creighton RA. The use of suture anchors in repair of the ruptured patellar tendon: a biomechanical study Am J Sports Med. 2006;34(9):1492-9.

12. Levy M, Goldstein J, Rosner M. A method of repair for quadriceps tendon or patellar ligament (tendon) ruptures without cast immobilization. Preliminary report. Clin Orthop Relat Res. 1987; 218(5):297-301.

13. Kasten P, Schewe B, Maurer F, Gosling T, Krettek C, Weise K. Rupture of the patellar tendon: a review of 68 cases and a retrospective study of 29 ruptures comparing two methods of augmentation. Arch Orthop Trauma Surg. 2001;121(10):578-82.

14. Bhargava SP, Hynes MC, Dowell JK. Traumatic patella tendon rupture: early mobilisation following surgical repair. Injury. 2004; 35(1):76-9.

15. Enad JG, Loomis LL. Primary patellar tendon repair and early mobilization: results in an active-duty population. J South Orthop Assoc. 2001;10(1):17-23.

16. Shelbourne KD, Darmelio MP, Klootwyk TE. Patellar tendon rupture repair using Dall-Miles cable. Am J Knee Surg. 2001; 14(1):17-20. 